This item was submitted to Loughborough's Research Repository by the author.

Items in Figshare are protected by copyright, with all rights reserved, unless otherwise indicated.

\title{
Electron and ion kinetics in a DC microplasma at atmospheric pressure
}

PLEASE CITE THE PUBLISHED VERSION

http://dx.doi.org/10.1109/TPS.2007.904827

PUBLISHER

(c) IEEE

LICENCE

CC BY-NC-ND 4.0

\section{REPOSITORY RECORD}

Choi, Jun, Felipe Iza, Jae Koo Lee, and Chang-Mo Ryu. 2019. "Electron and Ion Kinetics in a DC Microplasma at Atmospheric Pressure". figshare. https://hdl.handle.net/2134/3214. 
This item was submitted to Loughborough's Institutional Repository by the author and is made available under the following Creative Commons Licence conditions.

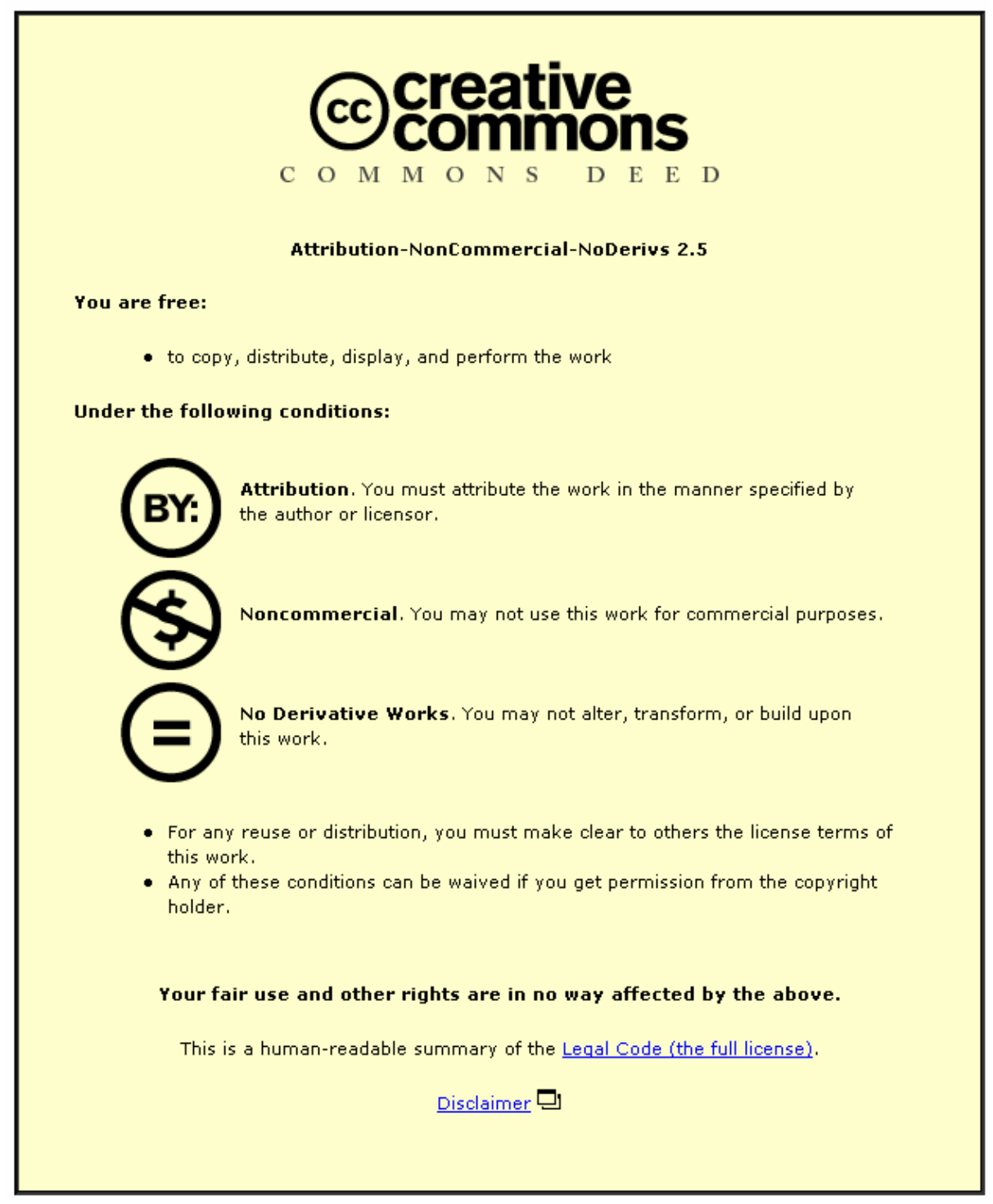

For the full text of this licence, please go to: http://creativecommons.org/licenses/by-nc-nd/2.5/ 


\title{
Electron and Ion Kinetics in a DC Microplasma at Atmospheric Pressure
}

\author{
Jun Choi, Felipe Iza, Member, IEEE, Jae Koo Lee, Senior Member, IEEE, and Chang-Mo Ryu
}

\begin{abstract}
The results of a particle-in-cell Monte Carlo collision (PIC-MCC) simulation of a direct current (dc) helium microplasma that operates at atmospheric pressure are presented. Electron and ion kinetic information that is not available from previous fluid studies is reported. Despite the high collisionality at atmospheric pressure, electrons are found to be in nonequilibrium. Similar to large-scale low-pressure dc discharges, the electron energy probability function (EEPF) in the bulk plasma presents three temperatures near the cathode, and it evolves into a bi-Maxwellian distribution as electrons approach the anode. The bi-Maxwellian character of the EEPF in the elastic energy region is not accounted for in fluid models, and as a result, PIC-MCC simulations predict a lower electron temperature than fluid models. The mean energy of ions that are impinging on the cathode is found to be significantly lower than in low-pressure discharges due to the large collisionality of the sheaths.
\end{abstract}

Index Terms-Atmospheric-pressure glow discharge (APGD), kinetic simulation, microdischarge, microplasma.

\section{INTRODUCTION}

$\mathbf{L}$ OW-TEMPERATURE plasma processing at atmospheric pressure has received growing attention in recent years for its potential economic and technological advantages. Along with the development of large-scale systems, novel highpressure microplasmas have also been developed. These miniaturized discharges enable new applications of plasmas in fields that range from displays and light sources to chemistry and biomedicine [1]. Despite the recent rapid developments, the physics of low-temperature high-pressure microplasmas are not fully understood.

Due to their reduced dimension, rapid transient behavior, high collisionality, and large space gradients, obtaining and interpreting space- and time-resolved diagnostics are very challenging tasks. These challenges hinder the study of the underlying physics. Since computer simulations can overcome the experimental challenges, they have become a valuable means to study microdischarges.

Most computational studies of atmospheric-pressure microdischarges that are found in the literature use fluid-based models [2]-[10]. Fluid models can provide a valuable descrip-

Manuscript received February 15, 2007; revised May 2, 2007. This work was supported in part by the Korean Science and Engineering Foundation Grant and by the Korean Ministry of Education through its Brain Korea 21 program.

J. Choi, F. Iza, and J. K. Lee are with the Department of Electronic and Electrical Engineering, Pohang University of Science and Technology, Pohang 790-784, Korea (e-mail: jkl@ postech.ac.kr).

C.-M. Ryu is with the Department of Physics, Pohang University of Science and Technology, Pohang 790-784, Korea.

Color versions of one or more of the figures in this paper are available online at http://ieeexplore.ieee.org.

Digital Object Identifier 10.1109/TPS.2007.904827 tion of the plasma. However, if the plasma is not in equilibrium and nonlocal effects are important, significant discrepancies between fluid and kinetic predictions can happen [11], [12]. Since the nonequilibrium character of high-pressure microplasmas is now widely recognized, a kinetic study is timely needed to validate fluid simulations and to obtain kinetic information that is not available with fluid models.

In this paper, a current-driven atmospheric-pressure helium microdischarge is simulated using a 1-D particle-in-cell Monte Carlo collision (PIC-MCC) model. This model can capture fundamental plasma physics that cannot be simulated with fluid models such as nonlocal plasma kinetics, collisionless heating, and striation formation [13]-[16]. The PIC-MCC simulation results are compared, when possible, with the fluid simulation results that are reported in [2]. The space evolution of the electron energy probability function (EEPF) is qualitatively equivalent to that encountered in low-pressure direct current (dc) discharges [17], [18]: it is bi-Maxwellian in the bulk plasma and presents a high-energy tail near the cathode region. While the high-energy tail that results from the acceleration of secondary electrons across the cathode fall can be incorporated in hybrid models [3], [6], [8], the bi-Maxwellian character of the EEPF in the elastic energy range is typically not accounted for. As a result, the mean electron energy that is obtained in the PIC-MCC simulations is significantly lower than that predicted by fluid simulations. This difference in electron temperature can significantly alter the equilibrium of reactions that are controlled by low-energy electrons (recombination, step ionization, dimer formation, etc). While $80 \%$ of the energy input to the plasma is dissipated by ions, the energy that is transferred to the cathode by ions is significantly limited by collisions in the sheath.

This paper is structured as follows. In Section II, a description of the physical device and the numerical model are presented. Simulation results are discussed in Section III, and the conclusions are drawn in Section IV.

\section{PhysicAl AND NumericAl Model}

The plasma source consists of two parallel plate electrodes that are separated by a 200- $\mu \mathrm{m}$ gap. A schematic of the device is shown in Fig. 1. The discharge is sustained by a constant current source, and the current density is fixed at $1 \mathrm{~A} / \mathrm{cm}^{2}$. The discharge is sustained in helium at atmospheric pressure.

The discharge is simulated using XPDP1 [19], which is a 1-D in space and 3-D in velocity (1d3 v) PIC-MCC code. Electrons and helium ions $\left(\mathrm{He}^{+}\right)$are tracked in the simulation, while neutral particles are assumed to be uniformly distributed in 


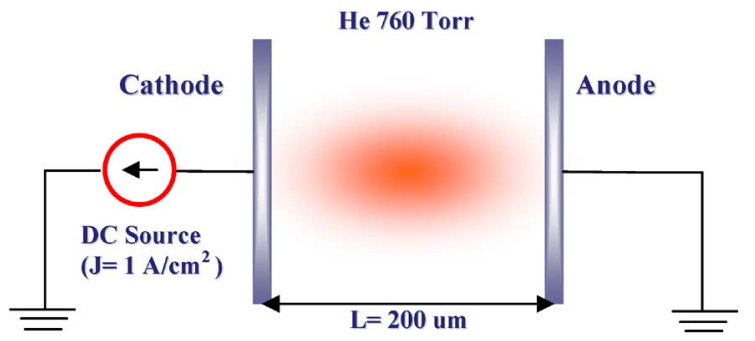

Fig. 1. Schematic of the parallel plate de microdischarge used in this work.

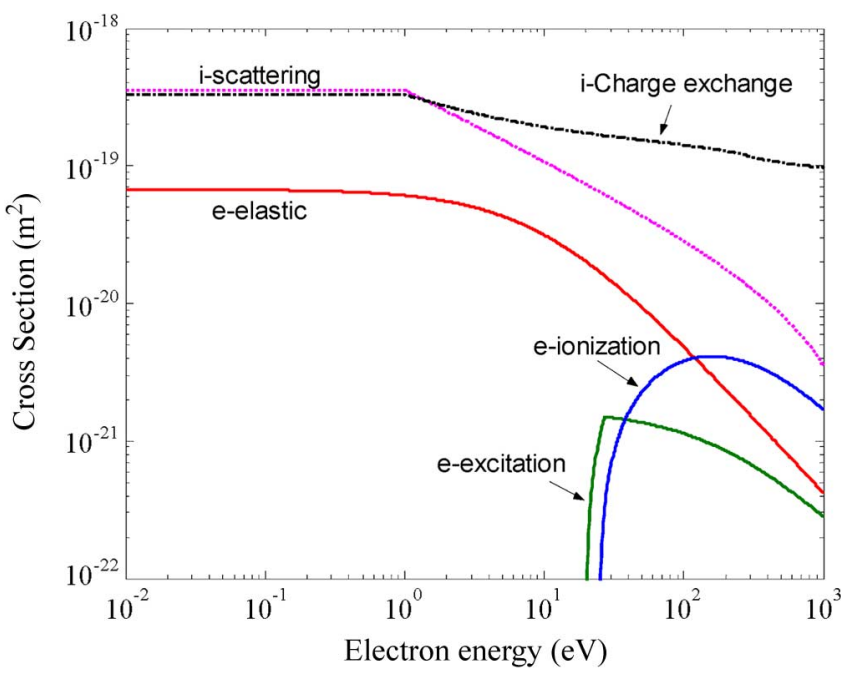

Fig. 2. Helium cross-sectional data set that is used in this paper.

space. The model accounts for elastic, excitation and ionization electron-neutral collisions, and for elastic scattering and charge exchange ion-neutral collisions. The cross-sectional data set that is used in this paper is shown in Fig. 2 [19].

DC discharges rely on secondary electron emissions from the cathode. A precise simulation of secondary electron emission processes should account for contributions from ions, metastables, hot neutrals, and photons [8]. In this paper, however, a constant ion-induced secondary electron emission coefficient (SEEC) of 0.1 is considered for simplicity. This is the same approach that is taken in fluid simulations, and the chosen value is the same as in [2], where simulation and experimental data were compared to determine the effective SEEC. Field emissions are not expected to be significant in our case, although they can become important in smaller gaps [20], [21].

\section{Simulation Results}

Fig. 3 shows the potential profile and the effective electron temperature that are predicted by the PIC-MCC model and compares them to the profiles that are obtained in [2] using a fluid model. The simulation conditions are similar in both studies, with voltage and current within the same order of magnitude: $250 \mathrm{~V}$ and $1 \mathrm{~A} / \mathrm{cm}^{2}$ in the present study and $230 \mathrm{~V}$ and $3.5 \mathrm{~A} / \mathrm{cm}^{2}$ in the fluid study [2]. Although the same qualitative picture is obtained with both simulation techniques, some differences can be noted.

The electron temperature reaches a maximum at the end of the cathode fall, and its value is significantly larger than in the

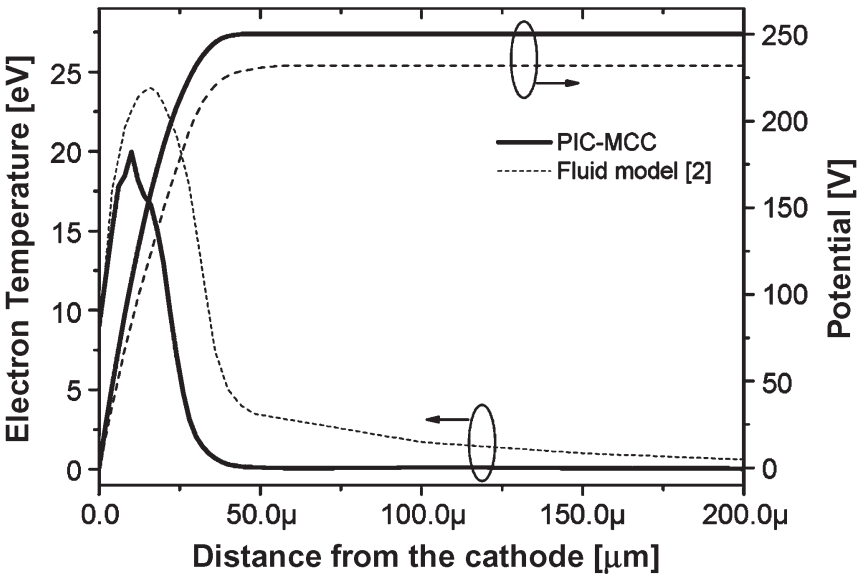

Fig. 3. Effective electron temperature and potential profiles in a $200-\mu \mathrm{m} \mathrm{dc}$ helium microplasma at atmospheric pressure. PIC simulations are presented with solid lines, and fluid simulations from [2] are presented with dotted lines.

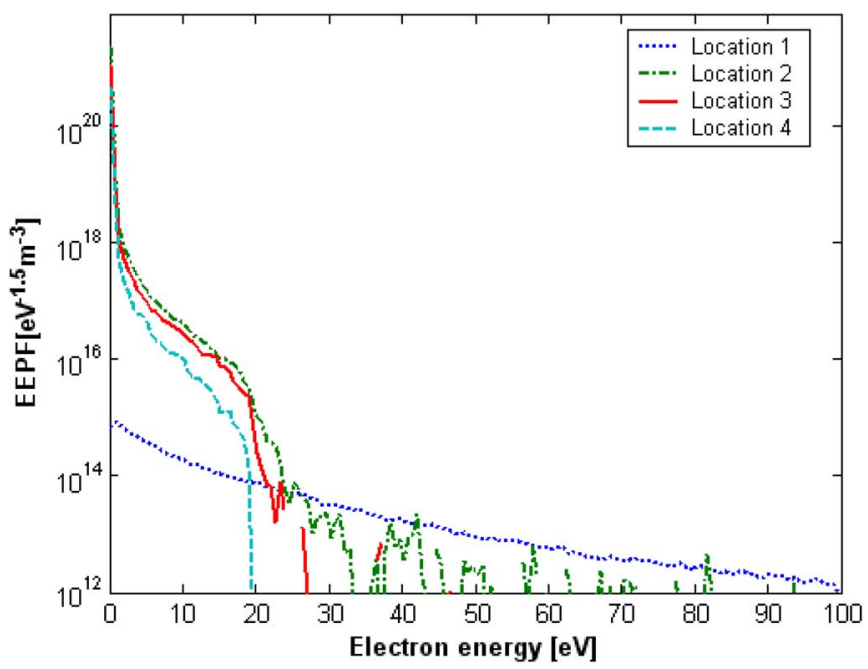

Fig. 4. EEPF at various locations in a dc helium microdischarge operated at atmospheric pressure. Locations $1-4$ correspond to $x=20,58,83$, and $136 \mu \mathrm{m}$ (see Fig. 6).

rest of the discharge. Fluid and PIC simulations agree quite well, and they predict a peak temperature of 24 and $20 \mathrm{eV}$, respectively. The decrease of the electron temperature as electrons move toward the anode is more abrupt in the PIC simulation, and a significantly lower temperature is observed in the bulk plasma. The faster decrease in the PIC simulations could be due, in part, to the omission of Penning ionization. Penning ionization can lead to the production of energetic electrons in the cathode region of the negative glow [2]. The electron temperature in the glow discharge that is predicted by the PIC model, however, is significantly lower than that predicted by the fluid model. The consistent lower electron temperature that is obtained in the PIC simulations is attributed to the assumption of a Maxwellian distribution in the fluid model. The difference may have also been favored by the lower neutral gas temperature that is used in this paper $(300 \mathrm{~K}$ versus $300 \mathrm{~K}-600 \mathrm{~K}$ in [2]).

As shown in Fig. 4, the EEPF is not Maxwellian, and three energy groups can be identified: low-energy electrons $(\varepsilon<$ $1 \mathrm{eV})$, mid-energy electrons $(1<\varepsilon<20 \mathrm{eV})$, and high-energy 


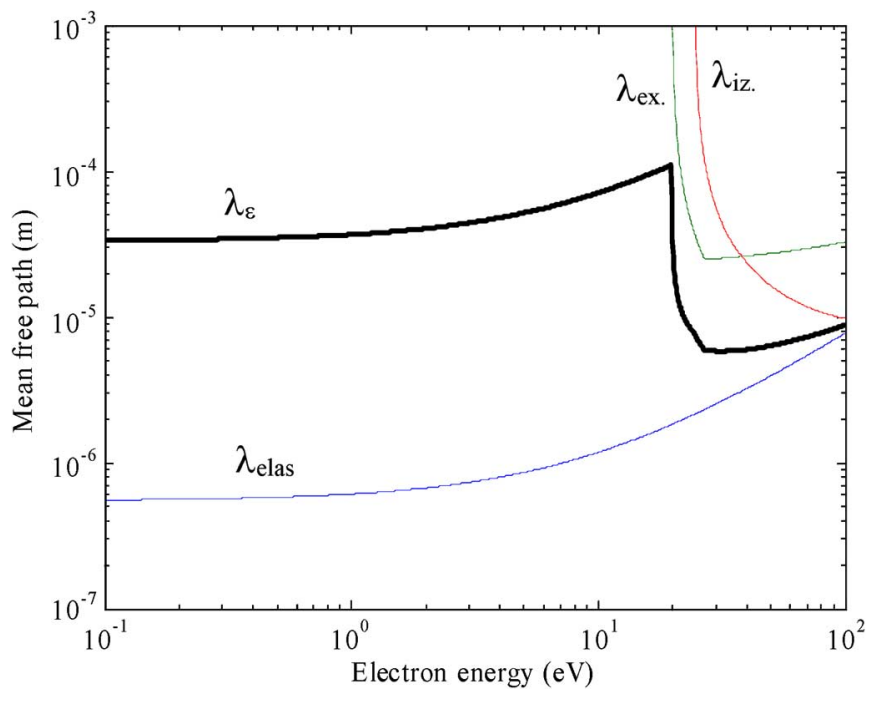

Fig. 5. Electron mean free path in a He discharge at atmospheric pressure for elastic $\left(\lambda_{\text {elas }}\right)$, excitation $\left(\lambda_{\text {ex }}\right)$, and ionization $\left(\lambda_{\mathrm{iz}}\right)$ collisions. $\left(\lambda_{\varepsilon}\right)$ is the energy relaxation length.

electrons $(\varepsilon>20 \mathrm{eV})$. Near the cathode, high-energy electrons are abundant due to the strong acceleration of electrons in the cathode fall. As electrons reach the bulk plasma, however, the high-energy tail quickly disappears because the electric field in the bulk plasma is much lower than in the sheath region. The energy relaxation length $\left(\lambda_{\varepsilon}\right)$ for electrons in the inelastic energy range is $<10 \mu \mathrm{m}$ (see Fig. 5). Thus, energetic electrons that are accelerated in the cathode fall barely penetrate into the bulk plasma, and most ionization takes place near the cathode region. A similar ionization profile is obtained with fluid models even if Penning and step ionization are included [2].

The EEPF in the bulk plasma is bi-Maxwellian due to the small electric field and the selective trapping of low-energy electrons by the ambipolar potential well. Mid-energy electrons $(1<\varepsilon<20 \mathrm{eV})$ that result from the avalanches in the cathode fall have a larger energy relaxation length than those in the inelastic energy range $(\varepsilon>20)$. As a result, these electrons can penetrate deeper in the discharge $\left(\lambda_{\varepsilon}=50 \sim 100 \mu \mathrm{m}\right.$; Fig. 5). These mid-energy electrons are less abundant than the low-energy ones. They are, however, important because they are not confined and they are the ones that support the electron current to the anode.

The EEPF profiles that are reported here are equivalent to those observed in low-pressure dc discharges. As in lowpressure discharges, a field reversal can take place in the negative glow near the cathode to preserve current continuity [2], [17]. For the conditions in our experiment, such field reversal occurs, but it is not appreciable in the potential profile that is shown in Fig. 3 due to the large voltage scale. This field reversal was also observed in fluid simulations [2].

Fig. 6 shows the electron and $\mathrm{He}^{+}$ion density profiles and compares them with those obtained with a fluid model in [2]. The sheath widths are comparable in both cases, and the peak densities are within the same order of magnitude. The density profiles, however, are significantly different. In the PIC simulation, the peak density is located near the cathode

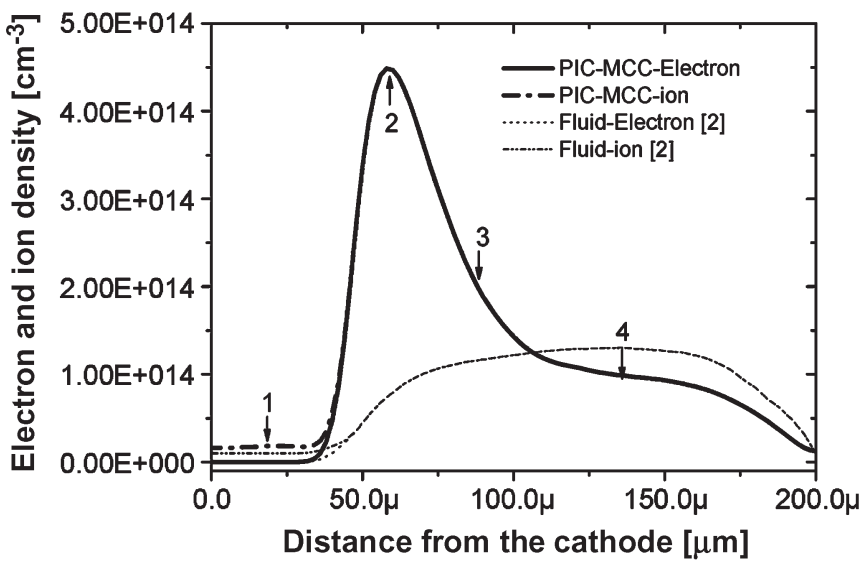

Fig. 6. Density profile in a dc helium microdischarge at atmospheric pressure. Arrows indicate the locations where the EEPFs that are reported in Fig. 4 are measured.

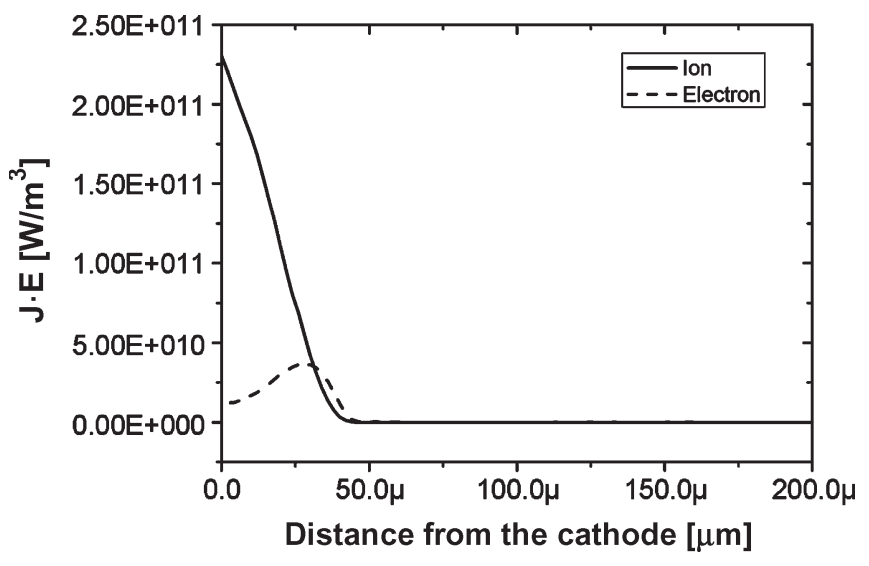

Fig. 7. Electron and ion power absorption $(J \cdot E)$ profiles in a dc helium microplasma at atmospheric pressure.

at $x \sim 60 \mu \mathrm{m}$, whereas in the fluid model, the peak density is located closer to the anode at $x \sim 140 \mu \mathrm{m}$. The larger plasma density that is near the anode region in fluid models is attributed to the presence of dimer ions $\left(\mathrm{He}_{2}{ }^{+}\right)$. Dimers are not included in the present PIC study. While $\mathrm{He}^{+}$ions are dominant near the cathode, fluid models [2], [4] indicate that dimer ions $\left(\mathrm{He}_{2}{ }^{+}\right)$ are the dominant positively charged species near the anode. The shift of the total plasma density to a position that is close to the anode that is reported in [2], however, seems counterintuitive and has not always been observed [4]. Such a shift may be due to ion production from metastable states that are not considered in the PIC simulation.

The electron and ion power absorption profiles are shown in Fig. 7. The power is measured as $J \cdot E$, where $J$ is the electron/ion current density and $E$ is the electric field. The profiles are characteristic of dc discharges, with most of the power being dissipated near the cathode region. The space-integral of the profiles that are shown in Fig. 7 indicates that $\sim 77.5 \%$ of the input power is absorbed by ions, while only $\sim 22.5 \%$ is absorbed by electrons. Similar numbers were reported in [2].

The energy distribution function of ions that are impinging on the cathode is shown in Fig. 8. The distribution is close to a Maxwellian distribution of temperature $\sim 1 \mathrm{eV}$. It is noted 


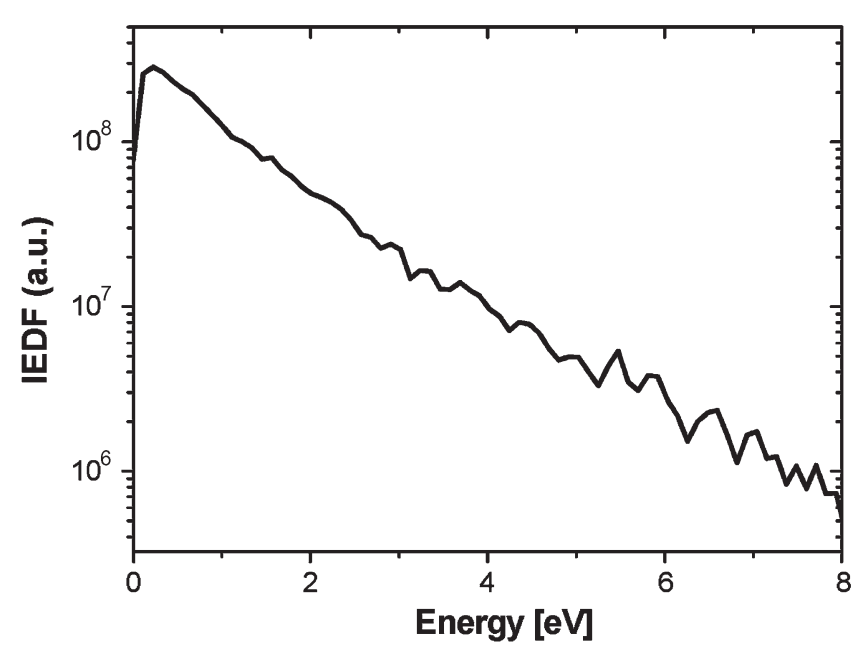

Fig. 8. Energy distribution function of ions impinging on the cathode.

that, despite the large potential drop across the cathode sheath $(\sim 250 \mathrm{~V})$, ions strike the cathode with a relatively low energy. This is due to the fact that the ion mean free path $(<0.1 \mu \mathrm{m})$ is much smaller than the sheath width $(\sim 30 \mu \mathrm{m})$, and therefore, ions lose most of their energy through collisions with the background neutral gas. While no fundamental difference is found between the EEPF of low-pressure and atmosphericpressure dc discharges, the mean energy of ions that strike the cathode is significantly lower at atmospheric pressure. This should result in a longer lifetime of the device, provided that they operate with the same current density.

Finally, it is noted that, due to the large collisionality at atmospheric pressure, the electron energy relaxation time is on the order of picoseconds for electrons in the inelastic energy range and of nanoseconds for electrons in the elastic energy range. As a result, conventional RF discharges that operate at 13.56 $\mathrm{MHz}(\tau \sim 74 \mathrm{~ns})$ can be seen as a succession of $\mathrm{dc}$ discharges that are operated at different voltages, and some similarities can be found between the EEPF in RF and dc discharges [22].

\section{CONCLUSION}

The results of a kinetic simulation of a de microplasma across a $200-\mu \mathrm{m}$ gap in helium at atmospheric pressure have been presented. The simulation results show that, despite the high collisionality, electrons are not in thermodynamic equilibrium, and as in low-pressure dc discharges, the assumption of a Maxwellian distribution that is used in fluid models is not precise. The EEPF in the bulk plasma evolves from a threetemperature distribution near the cathode to a bi-Maxwellian distribution near the anode. While hybrid models have been developed to account for the high-energy tail that results from avalanches in the cathode, the bi-Maxwellian character of the EEPF in the elastic energy range is not taken into account in fluid-based models. As a result, fluid simulations overestimate the electron temperature. This overestimation could affect the equilibrium condition that is predicted by fluid models, especially for reactions that are controlled by low-energy electrons, such as recombination, dimer formation, and step ionization.
The simulation results presented in this paper suggest that the electron kinetics in dc microdischarges at atmospheric pressure is the same as in large-scale low-pressure dc discharges. With regard to ion kinetics, however, the mean energy of the ions that arrive at the cathode is significantly lower at atmospheric pressure due to the large number of collisions that ions undergo as they transit the sheath.

\section{REFERENCES}

[1] K. H. Becker, K. H. Schoenbach, and J. G. Eden, "Microplasmas and applications," J. Phys. D, Appl. Phys., vol. 39, no. 3, pp. R55-R70, Feb. 2006.

[2] Q. Wang, D. J. Economou, and V. M. Donnelly, "Simulation of a direct current microplasma discharge in helium at atmospheric pressure," J. Appl. Phys., vol. 100, no. 2, p. 023 301, Jul. 2006.

[3] M. J. Kushner, "Modeling of microdischarge devices: Pyramidal structures," J. Appl. Phys., vol. 95, no. 3, pp. 846-859, Feb. 2004.

[4] P. S. Kothnur, X. Yuan, and L. L. Raja, "Structure of direct-current microdischarge plasmas in helium," Appl. Phys. Lett., vol. 82, no. 4, pp. 529-531, Jan. 2003.

[5] J. H. Seo and J. G. Eden, "Two-dimensional simulation of ac-driven microplasmas confined to 100-300 $\mu \mathrm{m}$ diameter cylindrical microcavities in dielectric barrier devices," J. Appl. Phys., vol. 100, no. 12, p. 123302 , Dec. 2006.

[6] J. J. Shi and M. G. Kong, "Cathode fall characteristics in a dc atmospheric pressure glow discharge," J. Appl. Phys., vol. 94, no. 9, pp. 5504-5513, Aug. 2003.

[7] J. J. Shi and M. Kong, "Evolution of discharge structure in capacitive radio-frequency atmospheric microplasmas," Phys. Rev. Lett., vol. 96 , no. 10, p. 105009 , Mar. 2006.

[8] Z. Donko, "Apparent secondary-electron emission coefficient and the voltage-current characteristics of argon glow discharges," Phys. Rev. E, Stat. Phys. Plasmas Fluids Relat. Interdiscip. Top., vol. 64, no. 2, p. 026401 , Aug. 2001.

[9] Y. Sakiyama and D. B. Graves, "Corona-glow transition in the atmospheric pressure RF-excited plasma needle," J. Phys. D, Appl. Phys., vol. 39, no. 16, pp. 3644-3652, Aug. 2006.

[10] A. Oda, T. Kimura, and Y. Oshikane, "A simulation of atmosphericpressure capacitive discharge in Ar," in Proc. 13th ACED, Oct. 2006.

[11] Electron Kinetics and Applications of Glow Discharges, ser. NATO Science Series: B, vol. 367, U. Kortshagen and L.D. Tsendin, Eds. New York: Springer-Verlag, 1998.

[12] H. C. Kim et al., "Particle and fluid simulations of low-temperature plasma discharges: Benchmarks and kinetic effects," J. Phys. D, Appl. Phys., vol. 38, no. 19, pp. R283-R301, Oct. 2005.

[13] G. Y. Park, S. J. You, F. Iza, and J. K. Lee, "Abnormal heating of low-energy electrons in low-pressure capacitively coupled discharges,' Phys. Rev. Lett., vol. 98, no. 8, p. 085 003, Feb. 2007.

[14] H. C. Kim and J. K. Lee, "Mode transition induced by low-frequency current in dual-frequency capacitive discharges," Phys. Rev. Lett., vol. 93, no. 8, p. 085 003, Aug. 2004.

[15] V. I. Kolobov, "Striations in rare gas plasmas," J. Phys. D, Appl. Phys., vol. 39, no. 24, pp. R487-R506, Dec. 2006.

[16] F. Iza, S. S. Yang, H. C. Kim, and J. K. Lee, "The mechanism of striation formation in plasma display panels," J. Appl. Phys., vol. 98, no. 4, p. 043 302, Aug. 2005.

[17] Y. P. Raizer, Gas Discharge Physics. Berlin, Germany: Springer-Verlag, 1991.

[18] G. J. Kim, F. Iza, and J. K. Lee, "Electron and ion kinetics in a micro hollow cathode discharge," J. Phys. D, Appl. Phys., vol. 39, no. 20, pp. 4386-4392, Oct. 2006.

[19] J. P. Verboncoeur, M. V. Alves, V. Vahedi, and C. K. Birdsall, "Simultaneous potential and circuit solution for 1D bounded plasma particle simulation codes," J. Comput. Phys., vol. 104, no. 2, pp. 321-328, Feb. 1993.

[20] M. Radmilovic-Radjenovic, J. K. Lee, F. Iza, and G. Y. Park, "Particle-incell simulation of gas breakdown in microgaps," J. Phys. D, Appl. Phys., vol. 38, no. 6, pp. 950-954, Mar. 2005.

[21] E. Hourdakis, G. W. Bryant, and N. M. Zimmerman, "Electrical breakdown in the microscale: Testing the standard theory," J. Appl. Phys., vol. 100, no. 12, p. 123 306, Dec. 2006.

[22] F. Iza, J. K. Lee, and M. G. Kong, "Electron kinetics in radio-frequency atmospheric-pressure microplasmas," Phys. Rev. Lett., vol. 99, no. 7, p. 075004,2007 


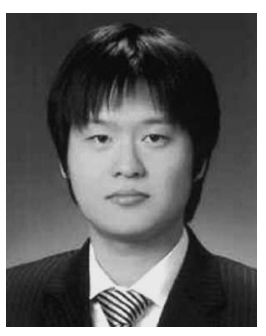

Jun Choi was born in Sokcho, Korea. He received the B.S. degree in electronic and electrical engineering from Chung-Ang University, Seoul, Korea, in 2003 and the M.S. degree in electronic and electrical engineering from Pohang University of Science and Technology, Pohang, Korea, in 2005, where he is currently working toward the Ph.D. degree.

His research interests include computational lowtemperature plasmas and atmospheric-pressure glow discharges.

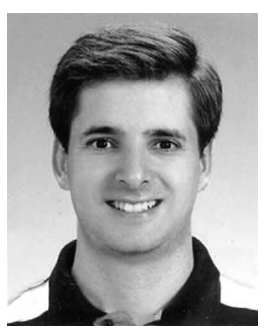

Felipe Iza (S'03-M'05) received the B.S. degree in engineering from the University of Navarra, San Sebastian, Spain, in 1997 and the M.S. and Ph.D. degrees from Northeastern University, Boston, MA, in 2001 and 2004, respectively.

From 1997 to 1999 , he was with the Centro de Estudios e Investigaciones Tecnicas de Guipuzcoa, San Sebastian. He is currently a Research Professor with the Department of Electronic and Electrical Engineering, Pohang University of Science and Technology, Pohang, Korea. His research interest includes experimental and computational low-temperature plasmas, with a special interest in atmospheric discharges and microplasmas.

Dr. Iza is a Fulbright alumnus and a member of the American Vacuum Society.

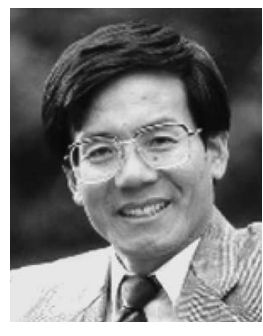

Jae Koo Lee (M'83-SM'02) received the Ph.D. degree from the University of California, Berkeley, in 1979.

From 1979 to 1989 , he was a Senior (later a Staff) Scientist on tokamak theory with the General Atomics, San Diego, CA. He is currently a Professor with the Department of Electronic and Electrical Engineering, Pohang University of Science and Technology, Pohang, Korea. He is currently on the Editorial Board of Journal of Physics D: Applied Physics and Plasma Processes and Polymer. His research interests include the theory and simulation of low-temperature basic/processing plasmas, fusion plasmas, and free-electron lasers.

Prof. Lee is a Fellow of the American Physical Society.

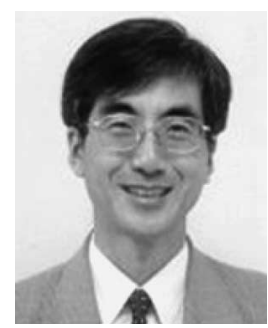

Chang-Mo Ryu received the B.S. degree in applied physics from Seoul National University, Seoul, Korea, in 1975 and the Ph.D. degree from Princeton University, Princeton, NJ, in 1983.

He is currently a Professor with the Department of Physics, Pohang University of Science and Technology, Pohang, Korea. His research interests include the theory and experiment of microplasmas, fusion plasmas, and space plasmas. 